FUB-HEP/94-5

\title{
Comment on Path Integral Derivation of Schrödinger Equation in Spaces with Curvature and Torsion
}

\author{
P. Fiziev* and H. Kleinert \\ Institut für Theoretische Physik, \\ Freie Universität Berlin \\ Arnimallee 14, D - 14195 Berlin
}

(January 7, 2018)

\begin{abstract}
We present a derivation of the Schrödinger equation for a path integral of a point particle in a space with curvature and torsion which is considerably shorter and more elegant than what is commonly found in the literature.
\end{abstract}

*Permanent address: Department of Theoretical Physics, Faculty of Physics, University of Sofia, Bull. 5 James Boucher, Sofia 1126, Bulgaria. Work supported by the Commission of the European Communities for Cooperation in Science and Technology, Contract No. ERB3510PL920264 


\section{INTRODUCTION}

When studying the historic paper on path integrals in spaces with curvature and torsion by DeWitt [1], or any of ist successors [2 [4], one may rightfully be frustrated by the tedious algebra involved in deriving the simple Schrödinger equation satisfied by a point particle in curved space. If torsion were to be admitted to the geometry, this derivation would become even more involved.

In this note, we want to point out that there exists a much shorter and more elegant derivation based on the use of nonholonomic coordinate transformations. By analogy with the physics of defects, these have recently been found to be an essential tool in predicting the correct path integral in such spaces [5-7]. The use of such transformations is very fundamental, and the transformation rules constitute a new quantum equivalence principle [5.6] which extends Einstein's famous classical principle to spaces with curvature and torsion, specifying the dynamical laws of both classical [8] and quantum physics [7].

Here we do not intend to convince the reader of the virtues of this principle but want to direct his attention upon a useful technical advantage of using such transformations: a drastic simplification of the above-mentioned derivation of the Schrödinger equation.

\section{SCHRÖDINGER EQUATIONS FROM PATH INTEGRAL}

Let us first recall the simple derivation of the Schrödinger equations from path integral of a free nonrelativistic particle of mass $M$ in euclidean space. The set of fluctuating paths in $D$ dimensions is parametrized by the time-dependent cartesian coordinates $x^{i}(t)(i=1, \ldots, D)$. The time axis is sliced into $N+1$ intervals $\left(t_{n}, t_{n-1}\right)(n=1, \ldots, N+1)$ of width $\Delta t$ and the positions $x^{i}\left(t_{n}\right)$ are denoted by $x_{n}^{i}$. If $\Delta x_{n}^{i}$ are the differences $x_{n}^{i}-x_{n-1}^{i}$, the time-sliced path integral has the measure

$$
\int d \mu_{x}^{\text {new }}:=\lim _{N \rightarrow \infty}\left[\prod_{n=2}^{N+1} \int \frac{d^{D}\left(\Delta x_{n}\right)}{(2 \pi i \hbar \Delta t / M)^{D / 2}}\right] \exp \left[\frac{i}{\hbar} \sum_{n=1}^{N+1} M \frac{\left(\Delta x_{n}^{i}\right)^{2}}{2 \Delta t}\right]
$$


Separating the last integral over $\Delta x_{N+1}$ from the others, the amplitude $\psi_{t}(x)$ at the time $t$ is seen to satisfy the recursion relation

$$
\psi_{t}(x)=\int \frac{d^{D}(\Delta x)}{(2 \pi i \hbar \Delta t / M)^{D / 2}} \exp \left[-\frac{M}{2 i \hbar \Delta t}\left(\Delta x^{i}\right)^{2}\right] \psi_{t-\Delta t}(x-\Delta x) .
$$

Expanding $\psi_{t-\Delta t}(x-\Delta x)$ in powers of $\Delta x$ and using the Gaussian integral formula

$$
\int \frac{d^{D} \Delta}{(2 \pi \varepsilon / a)^{D}} \exp \left[-\frac{a}{2 \varepsilon}\left(\Delta^{i} \Delta^{i}\right)\right]\left(1+b_{i} \Delta^{i}+b_{i j} \Delta^{i} \Delta^{j}+\ldots\right)=1+\frac{\varepsilon}{a} b_{i i}+\mathcal{O}\left(\varepsilon^{2}\right)
$$

one finds

$$
\begin{aligned}
\psi_{t}(x)=\int \frac{d^{D}(\Delta x)}{(2 \pi i \hbar \Delta t / M)^{D / 2}} \exp \left[i \frac{M}{2 \hbar \Delta t}\left(\Delta x^{i}\right)^{2}\right] \\
\quad \times\left[\psi_{t-\Delta t}(x)-(\Delta x)^{i} \nabla_{i} \psi_{t-\Delta t}(x)+\frac{1}{2}(\Delta x)^{i}(\Delta x)^{j} \nabla_{i} \nabla_{j} \psi_{t}(x)+\mathcal{O}\left((\Delta t)^{3 / 2}\right)\right] \\
=\psi_{t}(x)+\Delta t\left[-\partial_{t} \psi_{t}(x)+\frac{i \hbar}{2 M} \nabla^{2} \psi_{t}(x)\right]+\mathcal{O}\left((\Delta t)^{2}\right)
\end{aligned}
$$

In the limit $\Delta t \rightarrow 0$, this yields the Schrödinger equation

$$
i \hbar \partial_{t} \psi=-\frac{\hbar^{2}}{2 M} \Delta \psi
$$

where $\Delta \equiv \nabla^{2}$ is the Laplace operator.

In noneuclidean spaces, the derivation becomes complicated. For the sake of generality, we shall admit some kind of curvature and torsion. Let $q^{\mu}(t)(\mu=1, \ldots D)$ be the paths in such a general space $\mathcal{S}_{q}$. A nonholonomic transformation

$$
\dot{x}^{i}=e_{\mu}^{i}(q) \dot{q}^{\mu}
$$

maps them into a reference space $\mathcal{S}_{x}$ of $x^{i}$-vectors $(i=1, \ldots, D)$ with a euclidean metric. Under this transformation, the measure of path integration (11) goes over into [7]

$$
\int d \mu_{q}=\lim _{N \rightarrow \infty}\left[\prod_{n=2}^{N+1} \int \frac{d^{D}\left(\Delta q_{n}\right)}{(2 \pi i \hbar \Delta t / m)^{D / 2}} \frac{\partial\left(\Delta x_{n}\right)}{\partial\left(\Delta q_{n}\right)}\right] \exp \left(\frac{i}{\hbar} \Delta \mathcal{A}_{q}^{N+1}\right),
$$

where $\Delta \mathcal{A}_{q}^{N+1}$ is the time-sliced version of the classical action

$$
\mathcal{A}_{q}=\int_{t_{1}}^{t_{2}} d t \frac{m}{2} g_{\mu \nu}(q) \dot{q}^{\mu} \dot{q}^{\nu}
$$


of the system, evaluated along the classical orbits. The tensor $g_{\mu \nu}(q)=\sum_{i} e_{\mu}^{i}(q) e_{\nu}^{i}(q)$ is the metric with a nonzero Riemann curvature tensor $\bar{R}_{\mu \nu \lambda}{ }^{\kappa}$ derived from the covariant curl of the usual Christoffel symbols (Levi-Civita connection). The action $\Delta \mathcal{A}_{q}^{N+1}$ can be expanded around prepoint, midpoint, or postpoint in each time slice, with the latter being the most convenient one for the derivation of the Schrödinger equation [5,7]. It will be denoted by $\Delta \mathcal{A}_{>}^{N+1}$.

By a straight-forward but tedious generalization of the above euclidean derivation one finds in the space $\mathcal{S}_{q}$ the Schrödinger equation [1 50:0]

$$
i \hbar \partial_{t} \psi=-\frac{\hbar^{2}}{2 m} \Delta \psi
$$

where $\Delta \equiv D^{\mu} D_{\mu}$ is the Laplace operator in a general metric-affine space. This operator is related to the Laplace-Beltrami operator $\bar{\Delta} \equiv \bar{D}^{\mu} \bar{D}_{\mu} \equiv \sqrt{g}-1 \partial_{\mu} \sqrt{g} g^{\mu \nu} \partial_{\nu}$ by $D^{\mu} D_{\mu}=$ $\bar{\Delta}-2 S^{\mu} \partial_{\mu}$. The symbol $D_{\mu}$ denotes the covariant derivative with respect to the affine connection $\Gamma_{\mu \nu}^{\gamma}=e_{i}^{\gamma} \partial_{\mu} e_{\nu}^{i}$ with nonzero torsion $S_{\mu \nu}{ }^{\gamma}=\Gamma_{[\mu \nu]}^{\gamma}$, and zero Cartan curvature $R_{\mu \nu \lambda}{ }^{\kappa}=0$, whereas $\bar{D}_{\alpha}$ is the covariant derivative with respect to the Christoffel symbols $\bar{\Gamma}_{\mu \nu}{ }^{\kappa}=g^{\kappa \lambda}\left(\partial_{\mu} g_{\nu \lambda}+\partial_{\nu} g_{\mu \lambda}-\partial_{\lambda} g_{\mu \nu}\right) / 2$ with nonzero Riemann curvature $\bar{R}_{\mu \nu \lambda}{ }^{\kappa}$, and zero torsion $\bar{S}_{\mu \nu}{ }^{\gamma}=\bar{\Gamma}_{[\mu \nu]}{ }^{\gamma}=0$.

What makes the historic derivation [1 10 of the Schrödinger equation (9) tedious is the fact, observed first in Ref. [9], that one has to calculate the time-sliced action $\Delta \mathcal{A}_{>}^{N+1}$ up to fourth-order terms in the differences $\Delta q_{n}^{\mu}=q_{n}^{\mu}-q_{n-1}^{\mu}$, and the Jacobian $\partial\left(\Delta x_{n}\right) / \partial\left(\Delta q_{n}\right)$ up to the second-order. Since $\Delta q_{n}^{\mu}$ are of the order $\sqrt{\Delta t}$, all these terms contribute to first order in $\Delta t$, and thus to the Schrödinger equation. Instead of (3]), the derivation requires the more general formula

$$
\begin{aligned}
& \int \frac{d^{D} \Delta}{(2 \pi \varepsilon / a)^{D}} \sqrt{g}\left(1+b_{\mu} \Delta^{\mu}+b_{\mu \nu} \Delta^{\mu} \Delta^{\nu}+\ldots\right) \\
& \quad \times \exp \left[-\frac{a}{2 \varepsilon}\left(g_{\mu \nu} \Delta^{\mu} \Delta^{\nu}+G_{\mu \nu \lambda} \Delta^{\mu} \Delta^{\nu} \Delta^{\lambda}+G_{\mu \nu \lambda \kappa} \Delta^{\mu} \Delta^{\nu} \Delta^{\lambda} \Delta^{\kappa}+\ldots\right)\right] \\
& \quad=1+\frac{\varepsilon}{a}\left[b_{\mu \nu} g^{\mu \nu}-\frac{1}{2}\left(G_{\mu \nu \lambda \kappa}+G_{\mu \nu \lambda} b_{\kappa}\right) g^{\mu \nu \lambda \kappa}+\frac{1}{8} G_{\alpha \beta \gamma} G_{\mu \nu \lambda} g^{\alpha \beta \gamma \mu \nu \lambda}\right]+\mathcal{O}\left(\varepsilon^{2}\right) .
\end{aligned}
$$

Here $b_{\mu}, b_{\mu \nu}$, and $g_{\mu \nu}, G_{\mu \nu \lambda}, G_{\mu \nu \lambda \kappa}, \ldots$ are $\varepsilon$-independent coefficients, $g$ denotes the determi- 
nant of the metric det $\left\|g_{\mu \nu}\right\|$, and $g^{\mu_{1}, \ldots, \mu_{2 n}}$ are symmetric tensors formed from products of $n$ metric tensors: $g^{\mu \nu \lambda \kappa}=g^{\mu \nu} g^{\lambda \kappa}+g^{\mu \lambda} g^{\nu \kappa}+g^{\mu \kappa} g^{\nu \lambda}, \quad g^{\alpha \beta \gamma \mu \nu \lambda}=g^{\alpha \beta} g^{\gamma \mu \nu \lambda}+g^{\alpha \gamma} g^{\beta \mu \nu \lambda}+\ldots$. In the course of the calculations one encounters many cancelations, which make the final result (9) again very simple. There must be a derivation which reflects the simplicity of the final result from the beginning, and this is what we want to present now.

\section{NEW DERIVATION}

First we observe that the time-sliced action $\Delta \mathcal{A}_{q}^{N+1}$ which consists of the $\sum_{n=2}^{N+1} \Delta \mathcal{A}_{n}$ calculated along the classical trajectories is a simple Gaussian when expressed in terms of the velocities $\dot{q}^{\mu}$ in each time slice. The classical trajectories are described by the equation of motion

$$
\ddot{q}^{\mu}+\Gamma_{\nu \lambda}^{\mu} \dot{q}^{\nu} \dot{q}^{\lambda}=0
$$

This equations implies the conservation of the energy $E=g_{\mu \nu} \dot{q}^{\mu} \dot{q}^{\nu} / 2$. As a consequence, the short-time action is

$$
\Delta \mathcal{A}=\frac{m}{2} g_{\mu \nu} \dot{q}^{\mu} \dot{q}^{\nu} \Delta t=\frac{m}{2 \Delta t} g_{\mu \nu} \Delta \xi^{\mu} \Delta \xi^{\nu}
$$

where we have found it useful to introduce the vector quantities $\Delta \xi^{\mu}$ defined by $\Delta \xi^{\mu} \equiv \dot{q}^{\mu} \Delta t$ whose size is of the order of $(\Delta t)^{1 / 2}$, to have quantities comparable to the previous differences $\Delta x^{i}$. In (12), the velocities $\dot{q}^{\mu}$ as well as the metric $g_{\mu \nu}$ are calculated at the latest time $t_{n}$ in the interval $\left(t_{n}, t_{n-1}\right)$, to have the preferred post-point form (any time would give the same result, due to energy conservation).

An explicit functional relation between $\Delta \xi^{\mu}$ and $\Delta q^{\mu}$ is obtained by Taylor-expanding

$$
\Delta q^{\mu}=q^{\mu}\left(t_{n}\right)-q^{\mu}\left(t_{n-1}\right)=q^{\mu}\left(t_{n}\right)-q^{\mu}\left(t_{n}-\Delta t\right)=\dot{q}^{\mu} \Delta t-\frac{(\Delta t)^{2}}{2 !} \ddot{q}^{\mu}+\frac{(\Delta t)^{3}}{3 !} \ddot{q}^{\mu}+\mathcal{O}\left((\Delta t)^{4}\right)
$$

and using (11), which implies for the higher time derivatives

$$
\ddot{q}^{\mu}=-\Gamma_{\nu \lambda}^{\mu} \dot{q}^{\nu} \dot{q}^{\lambda}, \quad \ddot{q}^{\mu}=-\left(\partial_{\kappa} \Gamma_{\nu \lambda}^{\mu}-2 \Gamma_{\{\kappa \delta\}}^{\mu} \Gamma_{\nu \lambda}^{\delta}\right) \dot{q}^{\kappa} \dot{q}^{\nu} \dot{q}^{\lambda}
$$


where curly braces around indices indicate their symmetrization. Hence,

$$
\Delta q^{\mu}=\Delta \xi^{\mu}+\frac{1}{2 !} \Gamma_{\nu \lambda}^{\mu} \Delta \xi^{\nu} \Delta \xi^{\lambda}-\frac{1}{3 !}\left(\partial_{\kappa} \Gamma_{\nu \lambda}^{\mu}-2 \Gamma_{\{\kappa \delta\}}^{\mu} \Gamma_{\nu \lambda}^{\delta}\right) \Delta \xi^{\kappa} \Delta \xi^{\nu} \Delta \xi^{\lambda}+\ldots
$$

and this may be inverted to

$$
\Delta \xi^{\mu}=\Delta q^{\mu}-\frac{1}{2 !} \Gamma_{\nu \lambda}^{\mu} \Delta q^{\nu} \Delta q^{\lambda}+\frac{1}{3 !}\left(\partial_{\kappa} \Gamma_{\nu \lambda}^{\mu}+\Gamma_{\{\kappa \delta\}}^{\mu} \Gamma_{\nu \lambda}^{\delta}\right) \Delta q^{\kappa} \Delta q^{\nu} \Delta q^{\lambda}+\ldots
$$

Using this equation, we change the integration variables $\Delta q_{n}^{\mu}$ in formula (7) into $\Delta \xi_{n}^{\mu}$, and find the following measure for the path integral

$$
\int d \mu_{q}=\lim _{N \rightarrow \infty}\left[\prod_{n} \frac{d^{D} \Delta \xi_{n}}{\left(2 \pi i \hbar \Delta t_{n} / m\right)^{D / 2}} \sqrt{g\left(q_{n}\right)}\right] \exp \left[\frac{i}{\hbar \Delta t} \sum_{n=1}^{N+1} g_{\mu \nu}\left(q_{n}\right) \Delta \xi_{n}^{\mu} \Delta \xi_{n}^{\nu}\right] .
$$

This expression is related to the flat-space measure (11) by just a linear transformation. The reason for this is that in terms of the nonholonomic $x$-variables, the equation of motion (11) is trivial: $\ddot{x}^{i}=0$. Hence $\dot{x}^{i}=$ const, and

$$
\Delta x^{i}=\dot{x}^{i} \Delta t=e_{\mu}^{i} \dot{q}^{\mu} \Delta t=e_{\mu}^{i} \Delta \xi^{\mu}
$$

so that $\partial(\Delta x) / \partial(\Delta \xi)=\operatorname{det}\left\|e_{\mu}^{i}\right\|=\sqrt{g}$.

Using the simple measure (16), we immediately find for an amplitude $\psi_{t}(q)$ the recursion relation (again by removing the last slice from the product of integrals)

$$
\psi_{t}(q)=\int \frac{d^{D} \Delta \xi}{(2 \pi i \hbar \Delta t / M)^{D / 2}} \sqrt{g} \exp \left(-\frac{M}{2 i \hbar \Delta t} g_{\mu \nu} \Delta \xi^{\mu} \Delta \xi^{\nu}\right) \psi_{t-\Delta t}(q-\Delta q(\Delta \xi)) \text {. }
$$

Expanding the amplitude inside the integral in powers of $\Delta q^{\mu}$, and expressing these in terms of $\Delta \xi^{\mu}$ with the help of (14), we find

$$
\begin{gathered}
\psi_{t}(q)=\int \frac{d^{D} \Delta \xi}{(2 \pi i \hbar \Delta t / M)^{D / 2}} \sqrt{g} \exp \left(-\frac{M}{2 i \hbar \Delta t} g_{\mu \nu} \Delta \xi^{\mu} \Delta \xi^{\nu}\right) \\
\times\left[\psi_{t-\Delta t}(q)-\left(\Delta \xi^{\mu}+\frac{1}{2} \Gamma_{\nu \lambda}{ }^{\mu} \Delta \xi^{\nu} \Delta \xi^{\lambda}\right) \partial_{\mu} \psi_{t-\Delta t}(q)+\frac{1}{2} \Delta \xi^{\mu} \Delta \xi^{\nu} \partial_{\mu} \partial_{\nu} \psi_{t}(q)+\mathcal{O}\left((\Delta t)^{3 / 2}\right)\right] \\
=\psi_{t}(q)+\Delta t\left\{-\partial_{t} \psi_{t}(q)+\frac{i \hbar}{2 m}\left[g^{\mu \nu} \partial_{\mu} \partial_{\nu} \psi_{t}(q)-\Gamma_{\kappa}{ }^{\kappa \mu} \partial_{\mu} \psi_{t}(q)\right]\right\}+\mathcal{O}\left((\Delta t)^{2}\right) .
\end{gathered}
$$

After making use of the identity $g^{\mu \nu} \partial_{\mu} \partial_{\nu}-\Gamma_{\kappa}{ }^{\kappa \mu} \partial_{\mu}=D^{\mu} D_{\mu}$, this is precisely the Schrödinger equation (9). 


\section{COMPARISON WITH THE HISTORIC DERIVATION OF THE SCHRÖDINGER EQUATION IN SPACES WITH CURVATURE AND TORSION}

To compare the present derivation with the historic derivation in Refs. [1 [5,7], we must

calculate $\Delta \mathcal{A}$ as a function of $\Delta q^{\mu}$ up to fourth order terms using formulas (12) and (15). This yields

$$
\begin{gathered}
\Delta \mathcal{A}=\frac{m}{2 \Delta t}\left[g_{\mu \nu} \Delta q^{\mu} \Delta q^{\nu}-\bar{\Gamma}_{\mu \nu \lambda} \Delta q^{\mu} \Delta q^{\nu} \Delta q^{\lambda}+\frac{1}{3}\left(\partial_{\mu} \bar{\Gamma}_{\nu \lambda \kappa}-\frac{1}{4} \bar{\Gamma}_{\mu \nu \sigma} \bar{\Gamma}_{\lambda \kappa}{ }^{\sigma}\right) \Delta q^{\mu} \Delta q^{\nu} \Delta q^{\lambda} \Delta q^{\kappa}\right. \\
\left.+\frac{1}{3} S^{\sigma}{ }_{\mu \nu} S_{\sigma \lambda \kappa} \Delta q^{\mu} \Delta q^{\nu} \Delta q^{\lambda} \Delta q^{\kappa}+\mathcal{O}\left((\Delta q)^{5}\right)\right]
\end{gathered}
$$

The Jacobian $\partial(\Delta x) / \partial(\Delta q)$ is conveniently given in the exponential form

$$
\partial(\Delta x) / \partial(\Delta q)=\sqrt{g} \exp \left(i \Delta \mathcal{A}_{J}^{\prime} / \hbar\right)
$$

with an effective action

$\frac{i}{\hbar} \Delta \mathcal{A}_{J}^{\prime}=-\Gamma_{\{\mu \nu\}}^{\mu} \Delta q^{\nu}+\frac{1}{2}\left[\partial_{\{\mu} \Gamma_{\lambda \nu\}}^{\mu}+\frac{1}{3}\left(\Gamma_{\{\mu \kappa\}}^{\mu} \Gamma_{\{\lambda \nu\}}^{\kappa}-\Gamma_{\{\lambda \kappa\}}{ }^{\mu} \Gamma_{\{\nu \mu\}}{ }^{\kappa}\right)\right] \Delta q^{\lambda} \Delta q^{\nu}+\mathcal{O}\left((\Delta q)^{3}\right)$.

Inserting these expansions into the product of integrals (7) we obtain a large number of terms. Upon applying formula (10), most of these cancel each other, leading again to the Schrödinger equation (9).

The additional labor is the same as if we were to prove the obvious identity

$$
[\partial(\Delta x) / \partial(\Delta q)][\partial(\Delta q) / \partial(\Delta \xi)]=\partial(\Delta x) / \partial(\Delta \xi)=\sqrt{g}
$$

by writing $[\partial(\Delta x) / \partial(\Delta q)]$ as in (21) expanded via (22), and writing $\partial(\Delta q) / \partial(\Delta \xi)=$ $\exp \left(i \Delta \mathcal{A}_{J}^{\prime \prime} / \hbar\right)$ with

$$
\frac{i}{\hbar} \Delta \mathcal{A}_{J}^{\prime \prime}=\Gamma_{\{\mu \nu\}}^{\mu} \Delta \xi^{\nu}-\frac{1}{2}\left(\partial_{\{\mu} \Gamma_{\lambda \nu\}}^{\mu}-2 \Gamma_{\{\{\mu \kappa\}}{ }^{\mu} \Gamma_{\lambda \nu\}}{ }^{\kappa}+\Gamma_{\{\mu \lambda\}}{ }^{\kappa} \Gamma_{\{\kappa \nu\}}{ }^{\mu}\right) \Delta \xi^{\lambda} \Delta \xi^{\nu}+\mathcal{O}\left(\Delta \xi^{3}\right),
$$

which follows from (14). Inserting here (15) one may reexpress the right-hand side as a power series in $\Delta q$, and form the sum $\Delta \mathcal{A}_{J}^{\prime}+\Delta \mathcal{A}_{J}^{\prime \prime}$. The many terms in this sum all cancel each other, as required by (23). 
All such complications are avoided by working with the variables $\Delta \xi^{\mu}$ in which the exponential in (19) is a pure Gaussian. 


\section{REFERENCES}

[1] B. S. DeWitt, Rev. Mod. Phys. 29, 377 (1957)

[2] K. S. Cheng, J. Math. Phys. 13, 1723 (1972)

[3] H. Dekker, Physica, A103, 586 (1980)

[4] G. M. Gavazzi, Nuovo Cimento, A101, 241 (1989)

[5] H. Kleinert, Mod. Phys. Lett. A4, 2329 (1989)

[6] H. Kleinert, Phys. Lett. B 236, 315 (1990)

[7] H. Kleinert, Path Integrals in Quantum Mechanics, Statistics, and Polymer Physics, World Scientific Publ. Co. (1995)

[8] P. Fiziev and H. Kleinert, Variational Principle for Classical Particle Trajectories in Spaces with Torsion, hep-th/9503074, and Euler Equations for Rigid Body - A Case for Autoparallel Trajectories in Spaces with Torsion, hep-th/9503075.

H. Kleinert und A. Pelster, Lagrange Mechanics in Spaces with Curvature and Torsion, FU-Berlin preprint 1996;

The three papers can be read directly on the www, the first under http://www.physik.fu-berlin.de/ kleinert/kleiner_re219/newvar.html, the second with 219 /newvar.html replaced by 224 /euler.html, the third by $243 /$ varpr.html.

[9] S. F. Edwards, Y. V. Gulyaev, Proc. Roy. Soc. London, A279, 229 (1964) 